\title{
An Empirical View on Raising to Subject
}

\author{
Scott Grimm
}

Stanford University

This paper employs empirical methods to examine verbs such as seem, for which the traditional raising to subject analysis relates pairs of sentences which differ by taking an infinitival or sentential complement. A corpus-driven investigation of the verbs seem and appear demonstrates that information structure and evidentiality both play a determinate role in the choice between infinitival or sentential complementation. The second half of the paper builds upon the corpus results and examines the implications for the standard claims concerning these constructions. First, pairs of sentences related by the subject-to-subject raising analysis of verbs are often viewed as equivalent. New evidence from indefinite generic subjects shows that whether an indefinite generic subject occurs in the infinitival or sentential complement construction leads to truth-conditional differences. Further implications are explored for the claim that subjects of the infinitival variant may take narrow-scope: once various confounds are controlled for, the subject of the infinitival construction is shown to most naturally take wide-scope.

\section{Introduction}

The construction known as subject-to-subject raising, proposed for verbs such as seem or appear, relates pairs of sentences which differ by taking an infinitival or sentential complement. An example typical of those usually considered is shown in (1) (Davies and Dubinsky 2004, p. 3).

(1) a. Barnett seemed to understand the formula.

b. It seemed that Barnett understood the formula.

Despite the prominent role this analysis has played in generative syntax, the empirical behavior of pairs of sentences related by the raising to subject analysis is relatively under-studied from an empirical standpoint, especially compared to 
the amount of recent work on the genitive and dative alternations in English (see representative studies Rosenbach (2002) and Bresnan et al. (2007), respectively). This paper focuses on establishing an empirical benchmark for the pairs of sentences at issue in the raising to subject analysis.

A guiding intuition has been that such pairs of sentences are truthconditionally equivalent (Davies and Dubinsky 2004, p. 4), although this equivalence has recently become a subject of debate (see Lasnik 2003 for a denial of the equivalence and Boeckx 2001 for counter-arguments). In particular, it has been argued that the raising predicate in (1a), in parallel to (1b), has wide-scope over the situation designated by the proposition, which has served as motivation for analyses such as quantifier lowering (May 1977). Two other claims, which concern selectional behavior, are central to the raising analysis. First, a raising predicate uniformly selects for a proposition as its complement at the level of semantic selection, in the sense of, for instance, Grimshaw (1979), whether a raising predicate is followed by an infinitival or a clausal complement. Second, the raising predicate does not select for its subject, rather the subject is selected with respect to the proposition.

This paper will present empirical evidence that the behavior of verbs such as seem and appear is much more intricate than these standard claims allow for. While the pairs of sentences in constructed examples such as (1) often plausibly coincide in interpretation, data from actual language use manifest a rich set of differences in the use of one sentence type as opposed to the other, and at the same time demonstrate that none of the three claims above can be maintained unmodified. As the term "raising" presupposes a certain analysis of the constructions, I will avoid it in what follows, and refer to the two items as infinitival complementation (InfComp), as in (1a), and sentential complementation (SentComp), as in (1b), respectively.

The first three sections present results from a corpus study of the verbs seem and appear. In section 2, I demonstrate a distinction between the infinitival and sentential complement constructions in terms of information structure. The basic result is that the InfComp construction aligns with a topic-comment configuration, and the subjects of the InfComp are topics, while the SentComp is not so restricted, permitting structures not conforming to a topic-comment configuration. Section 3 examines a usage difference between the InfComp and SentComp in terms of evidentiality, demonstrating that the SentComp construction is strongly associated with indirect evidential readings, as opposed to the InfComp construction which is associated with direct evidential readings. Section 4 uses statistical modeling techniques to examine both information structure and evidentiality against other factors known to influence argument alternations. The latter half of the paper examines the implications of the empirical generalizations. Section 5 demonstrates that for sentences with 
indefinite generic subjects, whether the subject occurs in an InfComp or a SentComp construction leads to differences in truth conditions. Finally, I discuss the implications of the empirical results for the claim that seem always has widescope over a proposition, even within an InfComp construction, showing that once various confounds are controlled for, the subject of the InfComp most naturally takes wide-scope.

\section{Information Structure Constraints}

The information, or discourse, status of the referent of a noun, e.g. whether reference to an entity has been previously established or is somehow accessible to the hearer, has been held responsible for a large number of syntactic alternations, such as locative inversion or there-insertion (Ward et al. 2002). This section examines the influence of the information status of the subjects in both the InfComp and SentComp constructions.

\subsection{Measuring Information Status}

I tested the influence of information status on data gathered from the British National Corpus (BNC). I limited my study to two verbs: seem and appear. I extracted from the parsed BNC all occurrences of both appear and seem followed by to $+I N F$ or that + CLAUSE, from which I took a randomized sample of 200 occurrences for each pairing of verb and complement type, for a total data set of 800 instances. Later in the process, three instances of the InfComp and two of the SentComp were eventually discarded, as they were structurally inadequate, leaving a total of 795. For each of these instances, I automatically extracted the (embedded) subject, whose accuracy I verified later in the process.

I will first discuss the annotation scheme I used to measure the information status of the various subjects. After discussing the results of the corpus study, I will relate the findings to the notion of topicality in general, showing that these findings support considering the subject of the InfComp to be a topic.

\subsubsection{The Annotation Scheme of Nissim et al. (2004)}

For each sentence in my sample, I determined the status of the subject with respect to the discourse. I anchored this study in the annotation scheme implemented in Nissim et al. (2004). This scheme, however, was constructed to measure hearer-accessibility, which is restricted compared with the more general notion of topicality. Topicality covers not only accessibility of discourse elements from the hearer's perspective, but also from the speaker's perspective. 
For instance, hearer-accessibility considers specific indefinites, e.g. a certain $X$, as discourse new, since they are unknown to the hearer; however, specific indefinites are capable of serving as topics and are clearly accessible to and identified as such by the speaker. I will use Nissim et al. (2004) as a basis for the corpus work, and return to examining the discrepancy between hearer- and speaker-accessibility at the end of this section.

The scheme of Nissim et al. (2004) subdivides old, mediated and new information into more fine-grained categories: ${ }^{1}$

Old: A nominal entity is considered discourse old if it has been previously mentioned, if it is a generic pronoun, or if it is a personal pronoun referring to the dialogue participants. Nissim et al. (2004) establish the subtypes: identity (co-reference with previously mentioned entity), event, general (dialogue participants), generic, ident-generic (co-referential with a generic entity), relative (relative pronouns).

New: An entity is new if it has not been previously mentioned and is not otherwise accessible to the hearer.

Mediated: "Mediated entities have not yet been directly introduced in the dialogue, but are inferable from previously mentioned ones, or generally known to the hearer" (Nissim et al. 2004, p. 1024). Nine subtypes are specified: general (culturally known entities, e.g. the moon), bound (bound pronouns), part (entities which stand in a part-whole relation to a previously mentioned entity), situation (entities which stand in a part-whole relation to, or are "evoked" by, a previously mentioned situation), event ("whenever an entity is related to a previously mentioned VP" (ibid. p. 1024)), set (when an entity is a sub- or superset of a previously mentioned entity), poss (entities which stand in a possessed-possessor relation to a previously mentioned entity), function value ("entity refers to a value of a previously mentioned function" (ibid. p. 1024)), aggregation (a conjoined entity which refers to a previously mentioned individual entity, or entities).

\subsubsection{Results and Discussion}

Both the InfComp and SentComp constructions admit instances of all the different categories, yet there are distributional asymmetries. The results of the corpus study showed a clear association between the subjects of the InfComp construction and elements made accessible in the preceding discourse, i.e. discourse-linked material, while subjects of the SentComp construction permitted material that was not linked to the preceding discourse.

\footnotetext{
${ }^{1}$ See Nissim et al. (2004) for details and heuristics for the annotation procedure.
} 
This can be seen in table 1, which gives the totals for the occurrences of each construction by coarse-grained information status type. While both the InfComp and SentComp are relatively close in the number of mediated entities as subject, the SentComp has a greater proclivity toward discourse-new entities than the InfComp, and inversely concerning discourse-old entities. These distributional differences are statistically reliable $\left(\chi^{2}: p<.0001\right)$.

Table 1: Coarse-Grained Information Status Distribution

\begin{tabular}{|c|c|c|c|c|c|}
\hline & mediated & new & old & expletive & Total \\
\hline SentComp & 144 & 47 & 174 & 33 & 398 \\
\hline InfComp & 124 & 13 & 234 & 26 & 397 \\
\hline
\end{tabular}

Further analysis of the information status subtypes (see section 4) shows that genericity is an additional factor which correlates with the choice of complement. For both old-generic (generic pronouns/entities) and old-identgeneric (referring to previously mentioned generic entities) are primarily populated by instances of the SentComp construction.

\subsection{Information Status and Topicality}

The distribution of information status types across the SentComp and InfComp shows a compelling trend for elements linked to the preceding discourse to appear in the InfComp, with the exception of generics. Information status, however, belongs to the broader notion of topicality. In particular, since the information status notions used here are directed towards the information at the disposition of the hearer, this measurement was not able to capture ways in which the speaker may identify a nominal. In fact, most of the InfComps with discourse-new subjects, while hearer new, are clearly speaker-identified, and coincide with noun phrases which would still qualify as 'aboutness topics' (Reinhart 1981). For instance, (2) possesses a discourse new subject, David Liddle; however, this NP is the head of a relative clause, which would qualify it as specific and capable of being a topic (see discussion in Erteschik-Shir 2007, p. 48). Similarly, in (3), the employer's combinations does not appear in the preceding discourse, and it is difficult to relate it to anything else substantially enough to qualify for mediated, but the entity is prefaced by certain, typically indicative of a specific reading, and thus speaker-identified, which once again qualifies it as a topic.

(2) And indeed David Liddle, Director of Community Leisure in Avon, who is in charge of Avon County Libraries, seems to have taken this very line 
at the same seminar.

(3) Certain kinds of employers' combinations seem to have been hindered by antitrust legislation and this may help to explain the generally low level of organisation among employers.

Similarly, there were nominal entities which, by the principles of the annotation scheme, counted as hearer-new, since they had not been previously mentioned nor could be said to be explicitly evoked by the previous situation in the discourse, yet, these nominals stood in contrast to the preceding topic, and so qualified as a contrastive topic. The following passage illustrates this situation, where the topic is the Zostera species, a type of sea grass, and the author brings up a previously unmentioned entity, green algae, as a point of contrast.

(4) Although present in Langstone Harbour there are now no beds of Zostera species in either Sussex estuary, although these certainly existed in the early years of this century, and Z. angustifolia was recorded near the Hayling shore of Chichester Harbour as recently as 1963. But substantial beds of green algae are present and appear to have spread in recent years; they are probably continuing to do so.

The above subject of an InfComp, then, qualifies as a specialized type of topic, a contrastive topic (see Erteschik-Shir 2007).

There was one legitimate instance of completely new discourse entity, yet its occurrence is probably to be taken with a grain of salt. The example in (5) is the opening line of a chapter of a novel and most likely serves a specific literary function.

There was an explosion of blood and the nose seemed to burst.

As corpus results can often be biased by modality, e.g. written rather than spoken material, as well as particular annotators, I sought to independently verify the results of the annotation performed on the BNC. I examined the occurrences of seem in a version of the Switchboard Corpus (Godfrey et al. 1992) for which a portion was annotated for information status as part of the LINK project (based on Bresnan et al. 2002 and Zaenen et al. 2004). The annotation scheme used in the previous section is that which was developed for the LINK corpus, so the comparison was straightforward.

The number of occurrences of the SentComp construction was too few to draw any conclusions; however, the corpus yielded 45 occurrences of the InfComp construction which were marked for information status. The general 
picture established in section 2.1.2 emerged in the LINK corpus as well, the majority of occurrences were old (31), and the rest were mediated (14). Therefore, the findings from the LINK corpus, that subjects of the InfComp are linked to the preceding discourse and qualify as topics, concur with the findings of the corpus extracted from the BNC.

\section{Evidentiality}

One previously noted meaning difference between the InfComp and SentComp for raising verbs is that the "raised construction" appears to entail a perceptual experience of the "raised" element (Postal 1974, Langlacker 1995, and Asudeh \& Toivonen 2007 for copy-raising). Postal (1974, p. 358), building on Cantrall (1970), gives examples such as the following:

(6) Julius Caesar strikes me as honest.

(7) It struck me that Julius Caesar was honest.

The verbs considered by Postal differ from those that are central to the investigation, namely seem and appear, but the generalization holds for these forms as well:

(8) Julius Caesar appeared (appears) to be honest.

(9) It appears that Julius Caesar is (was) honest.

In the examples from Postal, there is a discrepancy in tense; however, even normalizing that, it would be difficult to place (8) in a context where some sort of perceptual experience was not at issue.

This observation concerning perceptual experience would appear to be on the right track, yet it is not clear what type of perceptual experience counts as sufficient to guarantee the semantic well-formedness of a given sentence. In particular, subjects of InfComps need not be concrete, i.e. perceptible, objects and may be abstract nouns, as in (10).

(10) The argument appeared to make sense. (BNC G17)

Accordingly, a broader notion than the perceptual experience constraint is needed, and here I will reorient the perceptual constraint to connect with the domain of evidentiality, which supplies a contrast between direct and indirect evidentiality.

Direct evidential marking is used when "the speaker has some sort of 
sensory evidence for the action or event he/she is describing" (de Haan, 2004, p. 314). This can include visual, auditory or other means of directly witnessing the action, entity or event under consideration. Supplanting the perceptual experience constraint with a direct evidential one provides the generality needed to account for uses such as (10); further, this move relates the subject constraint to a clear function which is frequently grammaticalized cross-linguistically.

In contrast, indirect evidentials "are used when the speaker was not a witness to the event but when he/she learned of it after the fact" (ibid. p. 314). This includes two main subcategories of inference, "used when the speaker draws an inference on the basis of available, physical, evidence", and quotatives, "used when the speaker has been told about the action or event by another person", i.e. a hearsay function (ibid. p. 314).

Recasting the perceptual experience observation as a direct evidential function of the InfComp leads one to expect that this function of the InfComp would stand in contrast to a function of the SentComp, with which it alternates. It follows that the SentComp should possess a function of marking indirect evidentiality. Indeed, corpus work reveals that marking inferences, one of the subfunctions of indirect evidentiality, is highly visible in occurrences of SentComp constructs. I now turn to the results of a corpus examination.

\subsection{Quantifying Evidential Marking}

The hypothesis that the InfComp is associated with direct evidential uses while the SentComp is associated with indirect evidential uses leads to a straightforward corpus method for determining whether the hypothesis holds. Since indirect evidentials are the marked member of the direct/indirect contrast, one would expect to find the SentComp construction overtly displaying its inferential function by reporting the source of evidence. As direct evidentials are the unmarked form, it is difficult to code for direct evidentiality in an objective manner.

I tested the evidentiality hypothesis on the corpus described in section 2.1. The corpus revealed a strong tendency for the SentComp to explicitly mark an inference based on evidence, one extremely clear example of which is (11).

(11) On this evidence, then, it would appear that the UK is a service economy.

When a token contained an explicit indicator of evidential source and/or inference, I counted the token as positive for indirect evidentiality. The most frequent indicator was explicit mention of the evidence upon which the judgment was made in a from-phrase, as shown in (12). Other frequent indicators were therefore, then and thus. If the context made it exceptionally 
clear that a conclusion was being drawn, for instance as in scientific textual contexts as exemplified in (13), I marked such instances as positive.

(12) It also seemed, from the feathers on the kitchen floor, that one of the pigeons had come down for a warm and had got too close.

(13) Experiments suggest that it has a fluid consistency and that there is movement of the liquid molecules within the membrane. It would also seem that some proteins are free to move laterally within the membrane.

The source of evidence was signaled by a wide variety of grammatical means. Table 2 summarizes the different indicators of evidential source found in the SentComp sentences along with the number of tokens for each in the corpus. As over a third of the SentComp sentences had some marking for inference and/or evidential source, the association between SentComp and indirect evidentials appears to be quite strong.

Table 2: Distribution of Indirect Evidential Markings

\begin{tabular}{ll} 
as & 2 \\
because & 2 \\
consequent & 10 \\
evidential source & 11 \\
for phrase & 5 \\
from phrase & 40 \\
given that & 2 \\
legal conclusion & 5 \\
on phrase & 5 \\
scientific conclusion & 15 \\
then & 12 \\
therefore & 19 \\
thus & 7 \\
other & 14 \\
\hline Total (out of 398 tokens) & 147
\end{tabular}

\subsection{Variability of Evidential Type for the InfComp}

The direct evidential/perceptual constraint interpretation is consistent with the majority of the InfComp occurrences; however, some instances of the InfComp show inferential uses as well, as in (14).

(14) Some expert clauses have referred the issue not just to one expert but to 
two experts and an umpire. From the cases, this seems to have been common practice in the nineteenth century: see for instance Re CarusWilson \& Greene (1886) 18 QBD 7, where there were two valuers and an umpire to value timber in a land sale. (BNC J6Y)

In the BNC corpus, I counted five instances of appear and one of seem as indirect evidential. In particular, when the complement is in the perfect, as in (14), the indirect evidential interpretation is found. Additionally, when the subject is not in the same location as the speaker, as in "Ed seems to be in his office", indirect evidential interpretation can arise.

There is a substantial range of types of evidentials between the extremes of direct visual evidence and abstract inference. For instance, inference based on first-hand evidence, which seems to be the usage in (14). Although evidentiality was operationalized above as a binary category, it is more properly viewed as a spectrum of multiple methods of observation and inference. Further work is needed to examine correlations between more specific types of evidential statements and complementation choice.

\section{Controlling for Multiple Factors}

At this point, two different strains of evidence have been presented that show a distributional asymmetry between the InfComp and SentComp constructions. Yet, it is increasingly understood that construction choice can be influenced by multiple factors, as argued, for instance, in the study of Bresnan et al. (2007) on the dative alternation. In order to examine whether other factors which have been known to have an influence on other constructions played a role here, I coded the data for two additional factors: subject length and nominal expression type, summarized below.

Subject Length Length has been long noted as an important factor in syntactic realization; for example, heavy NP shift places a longer constituent at the end of the clause (Behagel 1909, Wasow 2002). I measured the length of the subject, either of the matrix subject in the case of InfComps or the embedded subject in the case of SentComps, in number of words. In determining the final model, I also included the log of the number of words.

Nominal Expression Type The choice between the use of a pronoun and full noun phrase has been shown to affect syntactic realization, for instance in the case of the dative alternation (Green 1971, Bresnan et al. 2007). I divided the possible nominal expression types into the following categories PRONOUN (including definite, personal and reflexive pronouns, as well as demonstratives), 
DEFINITE, INDEFINITE (including phrases with the indefinite article as well as bare plurals), PROPER NAME, QUANTIFIER (such as most, few, any), RELATIVE PRONOUN, THERE, and VERBAL. As a factor in the eventual model, I additionally used a simplified metric coding for pronoun vs. non-pronoun.

Results and Discussion The influence of the above factors were examined using a generalized linear model, fitted to the data by using a stepwise model comparison, computed with the $\mathrm{R}$ statistical programming software. The following factors served as input to the model: length of the subject, the log of length of the subject, the coarse-grained information status types (old/mediated/new), the fine-grained information status subtypes, nominal expression type, (non-) pronoun and indirect evidential.

Four factors turned out to be significant, all of which increased the likelihood of the SentComp: INDIRECT EVIDENTIAL $(\mathrm{p}<.0001)$, the information status subtypes NEW $(\mathrm{p}<0.005)$, OLD-GENERIC $(\mathrm{p}<0.05)$, and OLD-IDENTGENERIC $(\mathrm{p}<0.005)$. The $\log$ of the subject length demonstrated a nonsignificant trend $(p=0.067)$, in the direction of the SentComp. Thus the information status categories and evidentiality are more predictive for these particular constructions than some of the better-known measures.

Having isolated the independent influence of topicality and evidentiality on the realization of the InfComp and the SentComp, it is incumbent upon a theory of verbs such as seem and appear to address why direct and indirect evidentiality functions should be associated with the InfComp and SentComp, respectively, rather than, for instance, the other way around. The previous sections, in fact, do suggest a motivation for this alignment. Direct evidential statements require an entity or event upon which the evidential statement is based, and this entity or event is presupposed with respect to the evidential statement. In the InfComp examples of seem and appear, the subject coincided with the entity that was the evidential source-for instance, in Ed seems to be tired, Ed serves as both the evidential source and the subject of the predication. If the evidential statement concerns a property of the entity which also serves as the evidential source, then this aligns with typical topic-comment structuresthe entity or event is backgrounded (topic) and the property predicated of the entity is asserted (comment), and thus the InfComp construction is appropriate. In contrast, for statements based on abstract inference, there is no particular entity that is presupposed with respect to the evidential statement, i.e. no entity is necessarily perceived prior to being able to make an abstract inference. Therefore, there is no ready backgrounded candidate to align with a topic, and thus, the InfComp is less likely to be appropriate. 


\section{$5 \quad$ Indefinite Generics}

Section 4 established that there was an asymmetry in the occurrence of generics with the two complementation types as generics occurred significantly more often with the SentComp. This section examines a further asymmetry in the acceptability of different types of generics in the InfComp construction. I will first differentiate the bare plural and the indefinite singular generics, and then examine how they behave within the context of the verbs and constructions at issue, showing that there are clear acceptability and truth-conditional differences dependent on whether an indefinite generic serves as the subject of seem or appear in the InfComp or the SentComp.

\subsection{Bare Plural and the Indefinite Singular Generics}

There is general agreement that one difference between the bare plural and indefinite singular generic constructions is that bare plurals may refer to a kind while indefinite singulars may not (see Krifka et al. 1995, Cohen 2001, and references therein). Sentences such as (15) are standard examples where something is predicated of a kind.

\section{Dinosaurs are extinct.}

When the generic is expressed by an indefinite singular, rather than a bare plural, the statement is infelicitous on the kind reading, as in (16).

\section{?A dinosaur is extinct.}

This contrast is taken as evidence that bare plural, but not indefinite singular, generics may denote kinds (Krifka et al. 1995, Cohen 2001).

A second difference between bare plural and indefinite singular generics is the different interpretations they allow. The bare plural allows for an inductivist or a normative (or definitional) reading, while the indefinite singular only permits a normative reading, suited to describing conventions and definitions (Cohen 2001). ${ }^{2}$

An inductivist use of a generic statement is true "iff sufficiently many relevant individuals in the domain of the generic satisfy the predicated property. (Cohen 2001, p. 194)" For instance, (17) is true if there are enough (relevant) kings to satisfy the sufficiently many requirement.

\footnotetext{
${ }^{2}$ The discussion here follows Cohen (2001), which builds on Carlson (1995).
} 
(17) Kings are generous.

Indefinite singular generics, if unmodified, do not permit the inductivist reading, as shown in (18).

(18) ?A king is generous.

If a statement such as (18) is modified so as to include quantification over situations, such statements can be rendered felicitous under the inductivist reading.

(19) A king is usually generous.

In contrast, the normative reading is most clearly in effect for statements of convention, such as (20) (Carlson 1995, p.225):

(20) Bishops move diagonally.

Normative generic sentences "do not get their truth or falsity as a consequence of properties of individual instances...instead, [they] are evaluated with regard to rules and regulations" (Cohen 2001, p. 194). If the generic sentence designates a rule which is in effect, then it is true, and otherwise false. Indefinite singular generics designate the normative reading, as shown in (21).

(21) A bishop moves diagonally.

On this account of generics, then, there is a truth conditional difference between the inductivist and normative readings. This truth conditional difference has been modeled in different ways. For instance, Papafragou (1996) in a modal treatment of generics distinguishes between bare plurals, which "require a realistic modal base", and indefinite generics, which may have modal bases which are not necessarily realistic. As an instance of the latter, Papafragou (1996) gives (22) as an example, which involves a deontic modal base which does not include the real world.

(22) A Christian is forgiving.

(22) clearly contrasts with (23), which on the preferred reading does say something about the real world. 
The contrast is summarized by Papafragou (1996, p. 17): "[(22)] does not make a statement about actual Christians but only about Christians in ideal (deontic) worlds; in fact, [(22)] does not even presuppose/imply the existence of Christians in the actual world, since the actual world does not belong to the modal base."

\subsection{Generics and Construction Choice}

Applying this distinction between bare plural and the indefinite singular generics to raising predicates reveals an asymmetry in acceptability of generics with the different complementation types. As shown in (24)-(25), indefinite singular generics with the InfComp, assuming neutral intonation and holding the generic interpretation constant, are unacceptable.

(24) a. A madrigal is polyphonic. (Cohen 2001)

b. ?A madrigal seems to be polyphonic.

(25) a. A pheasant lays speckled eggs. (Krifka et al. 1995)

b. ?A pheasant seems to lay speckled eggs.

Neither (24b) nor (25b) are interpretable while maintaining the definitional/normative reading. The more the generic statement hinges upon a convention, the worse these examples become:

(26) a. A bishop moves diagonally.

b. ?A bishop seems to move diagonally.

In contrast, when occurring in the SentComp, indefinite generics sentences maintain their definitional reading, as seen in (27)-(29).

(27) It seems that a madrigal is polyphonic.

(28) It seems that a pheasant lays speckled eggs.

(29) It seems that a bishop moves diagonally.

Indefinite singular generic subjects also appear freely as embedded subjects in naturally-occurring SentComp constructions, as witnessed in (30).

(30) It seems that a parent has a right to stalk their minor child, even if he is a 
teenager. (from BOARDS.LP.FINDLAW.COM)

InfComp constructions with indefinite generics which give a deontic reading of the type discussed for (22), repeated here as (31a) are also infelicitous. ${ }^{3}$ Once seems is inserted, the deontic force is no longer available.

a. A Christian is forgiving.

b. ?A Christian seems to be forgiving.
a. A gentleman opens doors. (Burton-Roberts 1977)
b. ?A gentleman seems to open doors.

The argumentation here should not be taken as saying that indefinite generics can under no circumstances be subjects of seem or appear, for of course they can, when their appearance is licensed by other factors. For instance, indefinite generics make excellent contrastive topics. Provided with the proper context, one can force acceptable readings of some of the above examples:

(33) A: Birds only lay pure white eggs.

B: A pheasant seems to lay speckled eggs.

These occur naturally as shown in (34), where health food shops is the preceding topic:

(34) What always strikes me about health food shops are the rows and rows of bottles and tablets. A greengrocer seems to be a much better source of natural products than such collections of distilled essences and the like. (HTTP://WWW.FALLACYFILES.ORG/ADNATURE.HTML)

Additionally, when embedded in a larger structure, namely when- or if- clauses or other modalized environments, the acceptability of indefinite generic subjects may also be affected, as the example in (35) shows.

(35) We will ignore the fact that a pronoun seems to be a special kind of noun, a noun that refers to a previously understood antecedent. (WWW.LLRX.COM/COLUMNS/GRAMMAR7.HTM)

\footnotetext{
${ }^{3}$ Thanks to Cleo Condoravdi for pointing out this class of examples to me.
} 
The claim here is that for the normative/definitional reading in neutral contexts, indefinite generic subjects occur without difficulty in the SentComp construction, but not in the InfComp construction.

Up until now, the focus has been on seem and appear, yet there are other verbs and adjectives analyzed as raising predicates, such as tend or likely. Parallel to seem and appear, the SentComp construction with likely does permit the normative reading. (Tend does not dispose of a SentComp construction.)

(36) It is likely that a bishop moves diagonally.

In contrast, indefinite singulars appear felicitously with both predicates:

(37) A bishop tends to move diagonally.

(38) A bishop is likely to move diagonally.

Yet, these predicates with the InfComp disallow the normative/definitional readings. To state that $X$ tends to $V$ implicates that this is not always the casewhich stands in contradiction to the normative/definitional reading, and similarly for likely.

(37) and (38), however, are not as difficult to accommodate as seem and appear with indefinite singular generics in the InfComp construction, for they do permit the inductivist reading. As was seen with (19), repeated here as (39), if an additional modifier is present, such as usually, indefinite singular generic sentences become acceptable under the inductivist reading, for such adverbs permit quantification over situations. A similar reading is available for tend, as shown in (40).

(39) A king is usually generous.

(40) A king tends to be generous.

Tend would appear to provide implicit quantification over situations, as would likely, which normally implies previous observations in order to declare whether something is likely or not. Thus, the subjects of predicates such as tend and likely are evaluated under the inductivist reading, where reference is made to "relevant individuals in the domain of the generic satisfy the predicated property." These subjects, then, are not an instance of non-referential, nontopical subjects with the InfComp, but rather of induced inductivist readings.

The above data leads to a generalization concerning the purported uniformity of propositional content selected by raising verbs discussed in section 1. The InfComp construction disallows the normative reading-indefinite 
singular statements in the InfComp are either infelicitous or induce an inductivist reading. No such constraint holds for the SentComp construction. Since the inductivist and normative readings correspond to different truth conditions, the InfComp and SentComp cannot be truth conditionally equivalent in all cases.

\subsection{Relating Generics and Evidentiality}

At this point, the question turns to why such a contrast should be present. One avenue of explanation involves the interaction between indefinite generics and topicality. According to Cohen (2001), the different readings of generics are directly related to the notion of topicality. A central requirement of topics is that they must refer to an individual of some sort. Bare plural subjects may refer to kinds in statements of direct kind predication, as well as individual instances of kinds in the inductivist readings. Kinds are individual entities (Carlson 1977), and so in both cases, there is a specific entity, kind or individual, which serves as a topic. Indefinite singular generics, however, do not refer to kinds and do not refer to individuals, as they do not permit the inductivist reading. On the normative reading, the subject need not be anchored in any particular referent. Thus, the subjects of indefinite singular statements do not refer to any specific individual, and so do not meet the requirement on topics.

If topicality is associated with the subjects of the InfComp, this predicts that indefinite generic subjects should be quite marked as subjects of the InfComp. This is indeed the case, as manipulating examples of indefinite singular generics shows. While this is a plausible line of explanation, the initial assumption that indefinite generics are non-topics is not universally shared, as the inventory of grammatical elements which are able to be topics is widely debated. For instance, the information structure annotation scheme elaborated in Götze et al. (2007) explicitly codes indefinite generics as aboutness topics.

At the same time, the type of evidentiality also contributes to the issue. In fact, the same acceptability patterns observed with indefinite generics by altering construction type (InfComp vs. SentComp) can be obtained by altering evidential type, as show in (41).

(41) a. I saw that a king is generous. (only inductive)

b. I heard that a king is generous. (inductive or normative)

This contrast opens the possibility that the (un)acceptability of indefinite generics is not directly due to topicality, but to the modal base given by the evidential reading at issue. Visual evidence is naturally constrained to be from a 
realistic modal base, while indirect/hearsay evidence has no such constraint, a difference in modal base requirements corresponds exactly to the difference in modal bases required for the inductive reading (realistic) and the normative reading (non-realistic/ideal) as discussed by Papafragou (1996).

Much more remains to be explored concerning the connections between genericity and evidentiality. Yet, if the observations above hold, then the behavioral differences of the InfComp and the SentComp can be tied to a meaning difference grounded in the forms of evidentiality associated with each type of construction.

\section{Scope}

The strong association between the subject of the InfComp and topicality has broad implications for possible scopal relations with raising predicates. In particular, a central claim in the raising analysis of predicates such as seem is that these predicates are able to take wide-scope with respect to the subject of the sentence, whether its form is the InfComp or SentComp, and this has been achieved through various mechanisms, such as "reconstruction" or "quantifier lowering". This section demonstrates that the facts concerning scoping are more complicated.

The actual data and observations concerning scoping are consistent with the topicality of InfComp subjects. As topics are by definition backgrounded and therefore referential, one would expect the subjects of the InfComp construction to be backgrounded, and therefore have wide-scope over the material in the VP. Thus, establishing that the subjects of InfComp are topics leads to a prediction that is in direct opposition to the claim that seem is able to be interpreted as taking wide-scope over its subject, regardless of its syntactic structure. This section assesses this prediction.

\subsection{Scopal Specificity}

The verbs which have canonically been considered to be "raising" verbs, seem and appear as well as adjectives such as likely and certain, have also been noted to be part of the class of lexical items known as non-factives (Kiparsky and Kiparsky 1970). In contrast to factives, which presuppose the content of the embedded clause, as in (42), non-factives have no such presupposition, as in (43).

(42) It is odd that it is raining. (factive)

presupposes that it is raining

(43) It is likely that it is raining (non-factive) 
does not presuppose that it is raining.

As non-factives permit an irrealis reading of their complement, they qualify as intensional verbs. One of the hallmarks of intensional verbs is that they permit both specific and non-specific readings of indefinites within their scope. The parade example is shown with the verb want in (44).

\section{(44) I want a book.}

$\Rightarrow$ There is a particular book such that I want it

(indefinite has wide-scope; the interpretation of the indefinite depends on the speaker's intended referent)

$\Rightarrow$ I want a book and, if there is one, any book will do

(indefinite has narrow-scope; the interpretation of the indefinite is bound by the intensional context introduced by want)

Since seem and appear are non-factive and induce irrealis contexts, both readings for indefinites should be available when within the scope of seem or appear. This is clearly true for the SentComp construction. In (45), the indefinite subject is non-specific with respect to the scope of appear; it is neither required to refer to a specific gun, nor to refer at all beyond the context established by appear.

(45) It appeared that a German S.P. gun had joined the snipers and was lobbing the occasional shell into the vicinity of the orchard. (BNC A61)

does not presuppose that there existed a particular German S.P. gun

The phrase could be felicitously continued by But, in fact, these shells came from a tank on the other side of ravine, and thus force the narrow-scope reading where a German S.P. gun does not refer outside of the context of appear.

Given the above observations, along with the interpretation traditionally claimed by the raising analysis, viz. that raising verbs always have wide-scope over the proposition, a testable prediction emerges: if raising verbs always have wide-scope, then indefinites should have both specific and non-specific irrealis readings available for both the InfComp and SentComp constructions. After discussing the notion of specificity in more depth, I will assess this claim.

\subsection{Specifying Specificity}

As specificity is a term used in a variety of senses, I begin with a few words about the sort of specificity intended here. The specific/non-specific distinction evoked by intensional predicates and non-factives has been termed scopal 
specificity (Farkas 1994, 2002). The difference between the two interpretations of the indefinite is determined by whether the indefinite is linked to the general context or to that context evoked by the intensional predicate: “...when scopally specific, the value of the indefinite is chosen from the domain of $w$, the world with respect to which the main clause is evaluated, while the value of scopally non-specific indefinites is to be chosen from the domain of worlds introduced by the predicate" (Farkas 1994, p. 4). In essence, the scopally non-specific reading is modal, determined by the set of possible worlds introduced by the predicate, while the scopally specific reading is actual, determined by prior discourse.

This sense of specificity must be kept distinct from others found in the literature, namely epistemic specificity and partitive specificity, exemplified in (46) from Fodor and Sag (1982) and (47) from Diesing (1992), respectively.

(46) a. A student in Syntax 1 cheated on the exam.

b. His name is John. (epistemic specific reading)

c. We are all trying to figure out who it was. (epistemic non-specific reading)

(47) a. There are some ghosts in this house. (partitive specific reading)

b. Some ghosts live in the pantry; others live in the kitchen. (partitive non-specific reading)

Epistemic specificity is distinct from scopal specificity - in (46a), no one doubts that there is a student who cheated, but there are just doubts about that student's identity, as the continuations of (46) show. As for partitive specificity, it too can be shown to be distinct from scopal specificity. Generally, a partitive use "denotes a member or subset of a familiar discourse group" (Farkas 1994, p. 8). Partitive non-specificity arises as to which member of the familiar set is being referred to. As in the case of epistemic specificity, there is no question that these members are in the world assumed by the speaker, in contrast to scopally non-specific readings. In the following, epistemic and partitive non-specifics are not at issue, it is the behavior of scopal (non-)specificity which will provide insight into whether InfComps and SentComps pattern similarly with respect to scope.

\subsection{Assessing Scopal Specificity}

One foundational fact about topics is that they are backgrounded, and therefore are referential with respect to the discourse. Section 2 established an association between the subject of the InfComp and topichood. Based on this finding, one would expect that the subject of the InfComp would align with the scopally 
specific reading and be unlikely to support the scopally non-specific reading. Naturally occurring examples provide evidence that the scopally specific/nonspecific distinction is employed in a manner consistent with these expectations, as demonstrated by a pair of discourses concerning governmental proceedings. The first in (48) shows the InfComp associated with the scopally specific reading while the second in (49) shows the SentComp construction used to indicate a scopally non-specific reading.

(48) The Senate adopted an extension of the provision with little attention to the issue, and the House opposed the measure, although on the only vote taken on it, a majority appeared to support continuing the measure. (The vote scorecard is accessible here [link].) However, the issue was obscured by the fact that some who voted against the effort to kill 245(i) insisted they simply did not want to tie the hands of the conference committee members who would decide the measure's fate.

(HTTP://WWW.FAIRUS.ORG/SITE/PAGESERVER?PAGENAME=IIC_IMMIGRATIO NISSUECENTERS326F)

In (48), the majority is actual, and can be verified by looking at the voting scorecard. However, what is undetermined is whether the majority intended to support the measure, or had an ulterior motive for voting as they did. Here, the subject of the InfComp is actual, i.e. scopally specific, while the complement of appears - the support for the measure - is merely potential. This differs from the SentComp structure in (49):

(49) A private member's bill to effect this change was brought forward in late February, 1925. Although there was some division of Conservative opinion, it appeared that a majority both of the Cabinet and of the backbenchers favoured either the acceptance of the bill or a Government measure doing roughly the same job in its place. Baldwin treated the matter with the utmost seriousness. He made a lot of soundings, appointed a special Cabinet committee to go into the subject, and held a special Cabinet to receive its report. (BNC EFN)

The context of (49) makes it is clear that the majority need not be actual- the votes have not taken place, and the main actor of the passage is busy sounding out the eventual voters.

Data such as the above would indicate that the subjects of the InfComp tend to be associated with the specific reading of indefinites, while subjects of the SentComp permit the scopally non-specific reading. Altering the SentComp 
example (45), repeated here as (50a), to (50b) shows that this distinction is at play.

(50) a. It appeared that a German S.P. gun had joined the snipers.

b. A German S.P. gun appeared to have joined the snipers.

The most natural reading, with neutral intonation, for $(50 \mathrm{~b})$ is that there is a specific German S.P. gun.

Similar contrasts are visible with other predicates traditionally analyzed as raising predicates, as shown for certain in (51). (51a) is a SentComp corpus example which permits a continuation explicitly denying the subject. Modulating the form to the InfComp as in (51b) once again makes such a continuation inaccessible.

(51) a. Nobody knows exactly who built South Luffenham, but it is almost certain that an architect called John Sturges supplied the drawings. (BNC AB4) ...although some deny the architect's existence.

b. Nobody knows exactly who built South Luffenham, but an architect called John Sturges is almost certain to have supplied the drawings.

...?although some deny the architect's existence.

\subsection{Lexical Semantic Confounds}

The previous section has added empirical support to the hypothesis that the SentComp and InfComp are distinguished in scopal properties just as one would expect if the subjects of the InfComp construction are topics, i.e. these subjects scope wide. Yet, one claim that is repeated throughout the literature, at least since Montague (1973), is that raising constructions permit scopally non-specific (i.e. narrow-scope) readings of the subject. The standard examples are of the following type:

(52) A cat seems to be in the garden. [embedded verb of existence]

(53) A train seems to be approaching. [embedded verb of appearance]

The intuition that these examples purportedly prompt is that no particular cat is actually in existence, and therefore, these sentences qualify as scopally nonspecific, and the entire phrase has a de dicto reading, equivalent to It seems that a cat is in the garden. While this intuition is relatively clear in the above 
examples, this could occur for independent reasons. The verbs used in such examples are from a restricted set-verbs of existence and appearance. When the phrases are minimally altered in the choice of the verb, the scopally nonspecific reading becomes far less accessible, if not impossible, as witnessed by (54) and (55), where the most natural readings involve a specific cat and a specific train.

(54) A cat seems to be sleeping in the garden.

(55) A train seems to be leaving.

The contrast between (52) and (54) and between (53) and (55) indicates that perhaps verbs of existence and appearance have a peculiar behavior which explains what has been claimed as the apparently exceptional behavior of the raising verbs. Indeed, these two classes of verbs share two peculiarities. First, most all transitive verbs entail that the subject exists prior to the event designated by the verb. For example, in John hit Bill, to perform the act of hitting, John must exist before the onset of the event. Verbs of existence and appearance, however, do not entail prior existence. Verbs of existence assert existence, and therefore do not entail prior existence. Verbs of appearance only entail that the argument exists at the location at the end of the event, but do not entail existence prior to the end of the event. Since seem modifies a VP, for statements such as seems to be arriving, seems indicates that the occurrence of the event is in question. As the existence of the subject is dependent on the occurrence of the event, it is unsurprising that the actuality of the subject is in question as well.

A similar confound is visible in examples containing predicates of negative existence, as exemplified in (56).

(56) Someone seems to be absent from class. (Boeckx 2001, p. 512)

While these examples do seem to have a preference for a narrow-scope reading, this has previously been shown to be independent of the 'raising' predicatethese verbs result in intensional object readings in the absence of any raising verb:

(57) A screw is missing from this TV set. (E. Bach reported in Dowty 1985)

In summary, while the intuition that the subjects of seem in the InfComp may take narrow-scope is indeed plausible for select sentences, these sentences may prefer such a reading for independent reasons. Given the strong association 
between the subjects of the InfComp and topicality for these verbs, it is unlikely that such readings are often generally available, as the evidence from corpus examples shows.

\section{Conclusion}

In section 1, some of the standard assumptions about the raising analysis were sketched, namely (i) a raising predicate uniformly selects for a proposition at the level of semantic selection, (ii) the InfComp and SentComp are taken to be interpretationally equivalent - in particular, in both the raising predicate has wide-scope over the situation designated by the proposition, and (iii) the raising predicate does not select for its subject, rather, the subject is selected with respect to the proposition. In light of the generalization concerning the information structure properties associated with the InfComp and SentComp structures, it is improbable that seem or other such predicates select for propositions in a simple or unified manner. Not just any proposition is felicitous in the InfComp formulation, but only those conforming to a topic-comment structure, in contrast to the less restrictive requirements of the SentComp. The second claim, that the InfComp and SentComp are meaning equivalent and that the raising predicate always has wide-scope, were brought into question in sections 5, which pointed to instances of non-equivalent truth conditions between the two structures, and 6, which demonstrated a consistent association between the subject taking wide-scope and the InfComp construction, as well as between the subject of seem or appear taking narrow-scope and the SentComp construction. The third claim, that the raising verbs in the InfComp do not select for their subject, must also be attenuated. While the subjects of the InfComp do not discriminate in terms of thematic content, it appears that such verbs, at minimum, do select for topics, and for seems and appear, the subjects of the InfComp are most often constrained to be direct evidential sources.

The results connect to higher-level theoretical points as well. The raising to subject analysis goes counter to the assumption of economy, common in linguistic theory: if the raised and non-raised constructions are equivalent, it is puzzling why this particular optionality of expression should exist. Why should a language bother to enforce two manners of saying the same thing? From the perspective of the data in this paper, this particular puzzle never occurs, for the two constructions simply differ in their associated meanings and uses.

The new empirical generalizations presented in this paper demonstrate that verbs such as seem and appear have a rich host of associations that can be used by in sophisticated ways. Incorporating these associations within the theoretical analysis of these verbs remains a challenge for future work. 


\section{Acknowledgements}

This work was presented at the 2009 Annual Meeting of the Linguistic Society of America, the Workshop on Linking of Sentential Arguments at the 2009 Deutschen Gesellschaft für Sprachwissenschaft, and at the Zentrum für Allgemeine Sprachwissenschaft in March, 2009, and I would like to thank the audiences of these conferences. This paper also greatly benefited from discussions with Eve Clark, Cleo Condoravdi, Hans-Martin Gärtner, Beth Levin, Asya Pereltsvaig, Ivan Sag, Kerstin Schwabe, Barbara Stiebels, and Tom Wasow. I would also like to thank in particular Beth Levin for acute comments on various drafts.

\section{References}

Asudeh, Ash \& Ida Toivonen (2007). Copy raising and perception. Unpublished ms.

Behagel, Otto (1909). Beziehungen zwischen Umfang und Reihenfolge von Satzgliedern. Indogermanische Forschungen, 25/110.

Boeckx, Cedric (2001). Scope reconstruction and A-movement. Natural Language and Linguistic Theory, 19:503-548.

Bresnan, Joan, Jean Carletta, Richard Crouch, Malvina Nissim, Mark Steedman, Thomas Wasow \& Annie Zaenen (2002). Paraphrase analysis for improved generation. LINK project, HRCR Edinburgh-CSLI Stanford.

Bresnan, Joan, Anna Cueni, Tatiana Nikitina \& Harald Baayen (2007). Predicting the dative alternation. In: Gerlof Bouma, Irene Krämer \& Joost Zwarts (eds.). Cognitive Foundations of Interpretation. Amsterdam: Royal Netherlands Academy of Science, 69-94.

Burton-Roberts, Noel (1977). Generic sentences and analyticity. Studies in Language, 1:155-196.

Cantrell, William (1970). Why do trains remind me of throwing up? Unpublished paper.

Carlson, Gregory (1977). Reference to Kinds in English. Ph.D. thesis, University of Massachusetts.

Carlson, Gregory (1995). Truth-conditions of generic sentences. In: Gregory Carlson \& Francis Pelletier (eds.). The Generic Book. Chicago: The University of Chicago Press, 224-237.

Cohen, Ariel. (2001). On the generic use of indefinite singulars. Journal of Semantics, 18:183-209.

Davies, William \& Stanley Dubinsky (2004). The Grammar of Raising and Control: A course in syntactic argumentation. Oxford: Blackwell.

de Haan, Ferdinand (2004). Semantic distinctions of evidentiality. In: Matthew Dryer, Martin Haspelmath, David Gil \& Bernard Comrie (eds.). World Atlas of Linguistic Structures. Oxford: Oxford University Press, 314-321.

Diesing, Molly (1992). Indefinites. Cambridge, Mass.: MIT Press. 


\section{Scott Grimm}

Dowty, David (1985). On Recent Analyses of the Semantics of Control. Linguistics and Philosophy, 8:291-331.

Erteschik-Shir, Nomi (2007). Information Structure: The Syntax-Discourse Interface. Oxford: Oxford University Press.

Farkas, Donka (1994). Specificity and scope. In: Léa Nash \& George Tsoulas (eds.). Langues et Grammaires 1. Paris.

Farkas, Donka (2002). Specificity distinctions. Journal of Semantics, 19:1-31.

Fodor, Janet \& Ivan Sag (1982). Referential and quantificational indefinites. Linguistics and Philosophy, 5:355-398.

Godfrey, John, Edward Holliman, \& Jane McDaniel (1992). Switchboard: Telephone speech corpus for research and development. In: ICASSP-92.

Götze, Michael, Thomas Weskott, Cornelia Endriss, Ines Fiedler, Stefan Hinterwimmer, Svetlana Petrova, Anne Schwarz., Stavros Skopeteas, \& Ruben Stoel (2007). Information structure. In: Stefanie Dipper, Michael Götze, \& Stavors Skopeteas (eds.). Working Papers of the SFB632, Interdisciplinary Studies on Information Structure (ISIS). Potsdam: Universitätsverlag Potsdam, 147-187.

Green, Georgia (1971). Some implications of an interaction among constraints. In: Papers from the Seventh Regional Meeting, Chicago Linguistic Society. Chicago: The University of Chicago, 85-100.

Grimshaw, Jane (1979). Complement selection and the lexicon. Linguistic Inquiry, 10:279326.

Kiparsky, Paul \& Carol Kiparsky (1971). Fact. In: Danny Steinberg \& Leon Jakobovits (eds.). Semantics. Cambridge: Cambridge University Press, 143-173.

Krifka, Manfred, Francis Pelletier, Gregory Carlson, Alice ter Meulen, Gennaro Chierchia \& Godehard Link (1995). Genericity: An introduction. In: Gregory Carlson \& Francis Pelletier (eds.). The Generic Book. Chicago: The University of Chicago Press, 1-124.

Langacker, Ronald (1995). Raising and transparency. Language, 71:1-62.

Lasnik, Howard (2003). Minimalist Investigations in Linguistic Theory. New York: Routledge Ltd.

May, Robert (1977). The Grammar of Quantification. Ph.D. thesis, MIT.

Montague, Richard (1973). The proper treatment of quantification in ordinary English. In: Jaakko Hintikka, Julius M.E. Moravcsik, \& Patrick Suppes (eds.). Approaches to Natural Language. Dordrecht: D. Reidel Publishing Co, 221-242.

Nissim, Malvina, Shipra Dingare, Jean Carletta, \& Mark Steedman (2004). An annotation scheme for information status in dialogue. In: Proceedings of the 4th Conference on Language Resources and Evaluation (LREC2004).

Papafragou, Anna. (1996). On generics. UCL Working Papers.

Postal, Paul (1974). On Raising. Cambridge, Mass.: MIT Press. 
Reinhart, Tanya (1981). Pragmatics and linguistics: An analysis of sentence topics. Philosophica, 27:53-94.

Rosenbach, Anette (2002). Genitive Variation in English. Conceptual Factors in Synchronic and Diachronic Studies. Mouton de Gruyter.

Ward, Gregory, Betty Birner \& Rodney Huddleston (2002). Information packaging. In: Rodney Huddleston \& Geoffrey Pullum (eds.). Cambridge Grammar of the English Language. Cambridge: Cambridge University Press, 1363-1447.

Wasow, Thomas (2002). Postverbal Behavior. Stanford: CSLI.

Zaenen, Annie, Jean Carletta, Gregory Garretson, Joan Bresnan, Andrew Koontz-Garboden, Tatiana Nikitina, M. Catherine O'Connor \& Thomas Wasow (2004). Animacy encoding in English: why and how. In: Donna Byron \& Bonnie Webber (eds.). The 2004 ACL Workshop on Discourse Annotation, 118-125. 